\title{
The effects of acute tryptophan depletion on costly information sampling: impulsivity or aversive processing?
}

\author{
M. J. Crockett • L. Clark • L. D. Smillie • T. W. Robbins
}

Received: 5 July 2011 / Accepted: 4 November 2011 /Published online: 19 November 2011

(C) Springer-Verlag 2011

\begin{abstract}
Rationale The neurotransmitter serotonin (5-HT) has been implicated in both aversive processing and impulsivity. Reconciling these accounts, recent studies have demonstrated that 5-HT is important for punishment-induced behavioural inhibition. These studies focused on situations where actions lead directly to punishments. However, decision-making often involves making tradeoffs between small 'local' costs and larger 'global' losses.

Objective We aimed to distinguish whether 5-HT promotes avoidance of local losses, global losses, or both, in contrast to an overall effect on reflection impulsivity. We further examined the influence of individual differences in subclinical depression, anxiety and impulsivity on global and local loss avoidance.

Methods Healthy volunteers $(N=21)$ underwent an acute tryptophan depletion procedure in a double-blind, placebocontrolled crossover design. We measured global and local loss avoidance in a decision-making task where subjects could sample information at a small cost to avoid making incorrect decisions, which resulted in large losses.
\end{abstract}

M. J. Crockett $\cdot$ L. Clark · T. W. Robbins

Behavioural and Clinical Neuroscience Institute,

University of Cambridge,

Cambridge, England, UK

M. J. Crockett $(\bowtie) \cdot$ L. Clark $\cdot$ T. W. Robbins

Department of Experimental Psychology,

University of Cambridge,

Downing Street,

Cambridge CB2 3EB, UK

e-mail: mollycrockett@gmail.com

L. D. Smillie

Psychological Sciences, The University of Melbourne,

Melbourne, Australia
Results Tryptophan depletion removed the suppressive effects of small local costs on information sampling behaviour. Sub-clinical depressive symptoms produced effects on information sampling similar to (but independent from) those of tryptophan depletion. Dispositional anxiety was related to global loss avoidance. However, trait impulsivity was unrelated to information sampling.

Conclusions The current findings are consistent with recent theoretical work that characterises 5-HT as pruning a tree of potential decisions, eliminating options expected to lead to aversive outcomes. Our results extend this account by proposing that 5-HT promotes reflexive avoidance of relatively immediate aversive outcomes, potentially at the expense of more globally construed future losses.

Keywords Serotonin · Punishment · Impulsivity · Behavioural inhibition · Tryptophan · Decision-making . Aversive processing $\cdot$ Depression $\cdot$ Anxiety

Growing evidence points to a selective role for the neurotransmitter serotonin (5-HT) in aversive processing (Soubrie, 1986; Deakin and Graeff, 1991; Tye et al., 1977; Boureau and Dayan 2011; Cools et al. 2011). Abnormal 5-HT function has been linked to psychopathologies associated with negative affect such as depression and anxiety (Neumeister, 2003; Harmer, 2008; Owens and Nemeroff, 1994; Graeff, 2002; Wise et al., 1972). Research in healthy animals and humans suggests that 5-HT is important for inhibiting behaviours that lead to punishment (Tye et al., 1977; Crockett et al., 2009; Geller et al., 1960; Stevens et al., 1969; Graeff and Schoenfeld, 1970; Wise et al., 1973; Thiébot et al., 1982; Cervo et al., 2000; Graeff, 2002). More recent work has refined this hypothesis by developing more sophisticated notions of how appetitive and aversive processes interact with 
activational and inhibitory processes (Boureau and Dayan 2011; Cools et al. 2011; Crockett et al., 2009). This work proposes that 5-HT plays a crucial role at the intersection of punishment and inhibition, translating aversive predictions into adaptive behavioural inhibition in a context-sensitive manner.

So far, studies in this area have focused on processing related to aversive outcomes resulting immediately from single actions (e.g., lever or button presses). However, many actions take place in the context of larger sequences, and in such contexts, an actor sometimes must choose whether to incur a small immediate loss in order to avoid a larger future loss. This tradeoff is central to many everyday decisions such as whether to purchase insurance, take exercise, or undergo mildly painful preventative medical procedures. In these kinds of decisions, aversive predictions occur at both the local level (expected pain of a dental cleaning) and the global level (expected pain of a root canal). There is evidence from the mental accounting literature that people segregate (rather than integrate) their evaluations of compound outcomes; in other words, a loss of $\$ 100$ plus a loss of $\$ 25$ is not psychologically equivalent to a loss of $\$ 125$ (Thaler, 1985). Moreover, research on construal level theory (Trope and Liberman, 2003) has shown that people represent psychologically distant events in an abstract, global fashion, whereas they represent psychologically proximal events in a concrete, local fashion. Construal level influences a range of processes important for decisionmaking (Dhar and Kim, 2007) such as probability estimation (Wakslak and Trope, 2009) and temporal discounting (Trope and Liberman, 2000). This indicates that people distinguish between global and local losses and that this distinction influences their decision-making. In the current study, we investigated to what extent 5-HT influences aversive processes at the local versus global level.

5-HT has also been implicated in impulsivity. However, attempts to specify the precise relationship between 5 -HT function and impulsivity have been only partly successful; 5-HT appears to play a role in some forms of impulsivity but not others (Robbins and Crockett, 2010; Evenden, 1999; Winstanley et al., 2004; Clark et al., 2005). 'Reflection impulsivity' is the tendency to make decisions before gathering sufficient or appropriate information (Kagan, 1966). This facet of pre-decisional impulsivity has received minimal research attention to date, and it is currently unclear whether tests of reflection impulsivity load primarily on the cognitive/risk-taking dimension or on the response inhibition dimension identified by impulsivity factor analyses (Reynolds et al., 2006; Meda et al., 2009). In rats, the effects of 5-HT manipulations on reflection impulsivity are mixed. Evenden (1999) reported a proimpulsive effect of the 5-HT2 antagonist ritanserin, an antiimpulsive effect of the 5-HT2 agonist DOI, both pro- and anti-impulsive effects of the 5-HT1A agonist 8-OH-DPAT, and no effects of the SSRI citalopram or the 5-HT1A antagonist WAY-100635. No study has yet examined the effects of manipulating 5-HT on reflection impulsivity in humans.

We examined the effects of acute tryptophan depletion (ATD), a dietary procedure that temporarily lowers brain 5HT levels (Biggio et al., 1974; Gessa et al., 1974; Moja et al., 1989; Ardis et al., 2009; Stancampiano et al., 1997; Carpenter et al., 1998), on behaviour in an Information Sampling Task (Clark et al., 2006) that allowed us to study global and local loss avoidance as well as reflection impulsivity. On each trial, subjects were instructed to accumulate as much information as they wished before making a decision. Information sampling predicted the probability of making a correct decision. Correct decisions won points that were exchangeable for money, and incorrect decisions lost points. We tested the effects of ATD on information sampling behaviour in two conditions. In the "Free" condition, there was no cost to sampling information. In terms of losses, subjects in this condition should only be concerned with losing money for incorrect decisions. Normatively, subjects should sample as much information as possible in order to avoid losing money at the end of the trial. In the "Costly" condition, subjects' potential winnings (if correct) decreased in direct proportion with the amount of information sampled. Thus, in this condition subjects must trade off the small immediate costs incurred by information sampling against the larger potential loss resulting from an incorrect decision.

Examining the effects of ATD on information sampling behaviour allowed us to distinguish to what extent 5-HT influences local aversive predictions, global aversive predictions, and reflection impulsivity. If 5-HT promotes avoidance of local losses, then ATD should increase information sampling in the Costly condition but have no effect on information sampling in the Free condition. Conversely, if 5-HT promotes avoidance of global losses, then ATD should increase information sampling in both the Free and Costly conditions. If 5-HT promotes loss avoidance at both global and local levels, then ATD should increase information sampling in the Free condition; whereas in the Costly condition, in which global and local loss avoidance are hypothesised to antagonise one another, the predicted effects of ATD are less clear. Finally, if 5-HT normally regulates reflection impulsivity, then ATD should increase reflection impulsivity, decreasing information sampling in both the Free and Costly conditions. As a further validation measure, we assessed the effects of individual variation in self-reported depressive symptoms (Beck Depression Inventory; Beck et al., 1996), dispositional anxiety (Behavioral Inhibition Scale; Carver and White, 1994) and impulsivity (Barratt Impulsivity Scale; Patton and Stanford, 1995). 


\section{Methods}

Participants

The experimental protocol was approved by the Norfolk Research Ethics Committee. Twenty-one healthy subjects (seven males; mean age 25.6 years) were screened for neurological and psychiatric disorders and gave written informed consent before participating in the study. Exclusion criteria included history of cardiac, hepatic, renal, pulmonary, neurological, psychiatric or gastrointestinal disorders, medication/drug use, and personal or family history of major depression or bipolar affective disorder. Participants were financially compensated for participating. After the screening interview, participants were assigned to receive either the tryptophan depleting drink (ATD) or the placebo mixture on the first session in a double-blind, approximately counterbalanced order. The Information Sampling Task (Clark et al., 2006) was administered as part of a larger cognitive assessment (reported separately; Crockett et al., 2008).

General procedure

Participants completed a battery of neuropsychological tests on two separate sessions, separated by at least 1 week. Participants were asked to abstain from food, alcohol and caffeine from midnight before each session. Testing sessions commenced in the morning (between 0830 and 1030 h). Upon arrival, participants completed self-report questionnaires, gave a blood sample, and ingested either the placebo or the tryptophan-depleting amino acid drink. After a resting period of approximately $5.5 \mathrm{~h}$ (to ensure stable and low tryptophan levels), participants completed a second self-report questionnaire, gave a second blood sample, and completed the test battery.

The Positive and Negative Affect Scale (PANAS; Watson et al., 1988) was administered on five separate occasions throughout the session. We analysed the difference in PANAS positive and negative affect scores from two time points: immediately before drink ingestion and immediately before the cognitive assessment.

Self-report measurements: depression, anxiety, and impulsivity

At the beginning of the first session (before the ATD procedure), we recorded self-report measures of depressive symptoms (Beck Depression Inventory (BDI); Beck et al., 1996), dispositional anxiety (Behavioral Inhibition Scale (BIS); Carver and White, 1994) and non-planning impulsivity (Barratt Impulsivity Scale, non-planning subscale (BARnp); Patton and Stanford, 1995). We focused on the non-planning subscale of the Barratt because it is directly relevant to the construct of reflection impulsivity and displays the highest correlations with decision-making tasks (de Wit et al., 2007; Zermatten et al., 2005).

The role of 5-HT in depression, anxiety, and impulsivity is undoubtedly complex. Although SSRIs, which enhance 5-HT function, are the first-line pharmacological treatment for depression (Neumeister, 2003; Owens and Nemeroff, 1994; Harmer, 2008), anxiety responds to SSRIs but also to benzodiazepines, which inhibit 5-HT function (Wise et al., 1972; Thiébot et al., 1982). Meanwhile, low 5-HT has been associated with impulsivity in personality disorders (Deakin, 2003; Dolan et al., 2001), but treating impulsivity in personality disorders with serotonergic drugs has had mixed success (Rinne et al., 2002; Zanarini et al., 2004). Comparing the effects of individual variation in these measures on information sampling with those of ATD can potentially inform an understanding of the role of 5-HT in these disorders. Importantly, BDI and BIS scores were not significantly correlated across subjects $(r=0.278, P=0.223)$ nor were BDI and BARnp scores $(r=0.045, p=0.846)$, or BIS and BARnp scores $(r=0.341, p=0.131)$.

\section{Acute tryptophan depletion procedure}

Tryptophan is the amino acid precursor of serotonin (5-HT), and dietary depletion of tryptophan causes a rapid decrease in brain 5-HT levels (Biggio et al., 1974; Gessa et al., 1974; Moja et al., 1989; Ardis et al., 2009; Stancampiano et al., 1997; Carpenter et al., 1998). In the ATD procedure, tryptophan was depleted by ingestion of a liquid amino acid load that did not contain tryptophan but did include other large neutral amino acids (LNAAs). Amino acid mixtures (prepared by SHS International, Liverpool, UK) were as follows:

ATD: L-alanine, $4.1 \mathrm{~g}$; L-arginine, $3.7 \mathrm{~g}$; L-cystine, $2.0 \mathrm{~g}$; glycine, $2.4 \mathrm{~g}$; L-histidine, $2.4 \mathrm{~g}$; L-isoleucine, $6 \mathrm{~g}$; Lleucine, $10.1 \mathrm{~g}$; L-lysine, $6.7 \mathrm{~g}$; L-methionine, $2.3 \mathrm{~g}$; Lproline, $9.2 \mathrm{~g}$; L-phenylalanine, $4.3 \mathrm{~g}$; L-serine, $5.2 \mathrm{~g}$; Lthreonine, $4.9 \mathrm{~g}$; L-tyrosine, $5.2 \mathrm{~g}$; and L-valine, $6.7 \mathrm{~g}$. Total: $75.2 \mathrm{~g}$.

Placebo: same as ATD, plus $3.0 \mathrm{~g}$ of L-tryptophan. Total: $78.2 \mathrm{~g}$.

For females, $20 \%$ reductions in the above quantities were used to account for lower body weight. The drinks were prepared by stirring the mixture and lemon-lime flavouring into $200 \mathrm{ml}$ tap water.

Information Sampling Task

The task was programmed in Microsoft Visual Basic 6.0 and administered on a touch-sensitive monitor. Test instructions (see "Appendix 1") were read aloud to the subject before a 
single practice trial. After practice, the subject was encouraged to ask any questions before starting the main task. On each trial (Fig. 1), subjects were presented with a $5 \times 5$ matrix of grey boxes, with two larger coloured panels at the foot of the screen. Touching a grey box caused the box to open (immediately) to reveal one of the two colours. The task was to decide the box colour in the majority on the board. The ratio of the two colours varied across trials from 8:17 to 12:13. Two pseudorandomised sequences were created as parallel forms and subjects were randomly assigned to either sequence on their first session. Opened boxes remained open for the duration of the trial. Subjects were able to open the boxes at their own rate, and the instructions emphasised that the subject could open as many boxes as they wished before making their decision.

Upon touching one of the coloured panels, the remaining boxes were uncovered and a feedback message "Correct! You have won $[x]$ points" or "Wrong! You have lost 100 points" was presented for $2 \mathrm{~s}$. The length of the inter-trial interval was adjusted, based on the response time for each trial, to establish a minimum duration of $30 \mathrm{~s}$ per trial. This feature was inserted to counteract delay-averse responding. During this delay, the current points total was presented centrally.

Subjects completed 10 trials in each of two conditions, with condition order counterbalanced across subjects. In the Free condition (previously called the 'Fixed Win' in Clark et al., 2006), the subject won or lost 100 points on each trial, irrespective of the number of boxes opened. In the Costly condition (previously called the 'Decreasing Win' condition), the available win decreased from 250 points in 10 point steps with every box opened (e.g., responding correctly after opening 12 boxes would yield 130 points). In the Costly condition, an incorrect decision lost 100 points, regardless of the number of boxes opened. Critically, the amount to be potentially won or lost on each trial was displayed in the upper left corner of the screen (Fig. 1). The only difference between the Free and Costly conditions was that the amount listed next to "Win" stayed fixed at 100 points in the Free condition but decreased by 10 with each box opened in the Costly condition.

To assess our critical dependent measure, the degree of information sampling in each condition, we calculated the probability of the subject being correct at the point of decision $[P($ correct $)]$. Under some circumstances, the number of boxes opened provides a limited index of the information available. For example, if the subject opens 20 boxes, these might be distributed 10 yellow and 10 blue $[P$ $($ yellow $)=0.5]$ or 15 yellow and 5 blue $[P($ yellow $)=1.0]$. The $P$ (correct) equation precisely quantifies the extent of information revealed on a trial-by-trial basis, although this variable is invariably correlated with the number of boxes opened. $P$ (correct) is calculated with the formula:

$P($ Correct $)-\frac{\sum_{k-A}^{z}\left(\begin{array}{l}z \\ k\end{array}\right)}{2^{z}}$

where $Z=25-$ (number of boxes opened), and $A=13-$ (number of boxes of the chosen colour). For example, if a

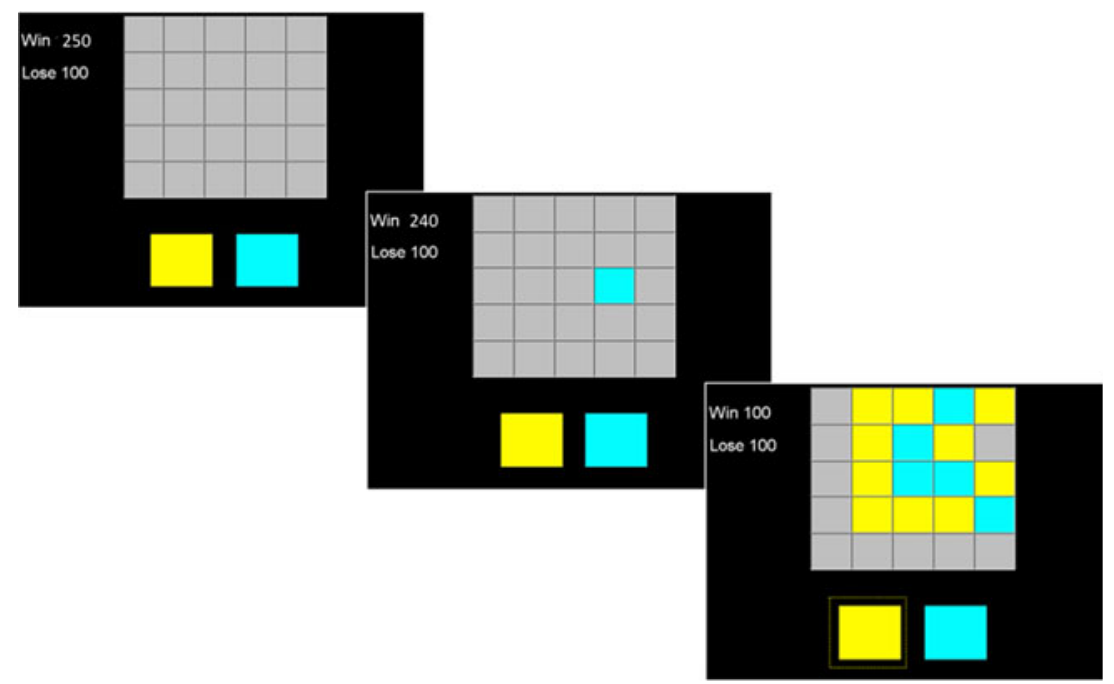

Fig. 1 Screen display for the Information Sampling Task (Costly condition). Each trial commenced (left) with the presentation of 25 grey boxes arranged in a $5 \times 5$ matrix, covering a random assortment of two colours of squares; these two colours were displayed on panels at the foot of the screen. When the subject touched a box (middle), it opened to reveal its colour. Subjects were instructed to decide which of the two colours was in the majority on the board. They were told they could open as many boxes as they wished and to touch the corresponding panel at the foot of the screen (bottom right) once they had decided which colour was in the majority. The amount to be won or lost on the current trial was displayed in the upper left corner; in the Costly condition, the "Win" amount decreased by 10 with each box opened. Reprinted from Biological Psychiatry, vol. 60, L. Clark et al., Reflection impulsivity in current and former substance users, p. 516, Copyright (2006), with permission from Elsevier 
decision is made toward yellow after opening 10 boxes ( 8 yellow, 2 blue), then $Z=25-10=15, A=13-8=$, and $P($ correct $)=[15 ! /(5 ! \times 10 !)+15 ! /(6 ! \times 9 !)+\ldots+15 ! /$ $b(15 ! \times 0 !)] / 2^{15}=0.94$.

General task performance was indexed by the number of correct judgments in each condition and total number of points scored. We also assessed the average box opening latency per trial (i.e., the amount of time between successive box openings).

\section{Data analysis}

\section{Biochemical measures}

Blood samples $(10 \mathrm{ml})$ were analysed to determine the total plasma TRP level and the TRP: $L$ LNAA ratio. This ratio was calculated from the serum concentrations of total tryptophan divided by the sum of the large neutral amino acids (tyrosine, phenylalanine, valine, isoleucine, and leucine) and is important because the uptake of TRP in the brain is strongly associated with the amounts of other competing LNAAs due to non-specific transport across the blood-brain barrier. Venous samples were taken in lithium heparin tubes and stored at $-20^{\circ} \mathrm{C}$. Plasma TRP concentrations were determined by an isocratic high performance liquid chromatography (HPLC) method of analysis. Plasma proteins were removed by precipitation with $3 \%$ trichloroacetic acid and centrifugation at $3,000 \mathrm{rpm}, 4^{\circ} \mathrm{C}$ for $10 \mathrm{~min}$ and then pipetted into heparin aliquots. An aliquot was diluted in mobile phase before injection onto the HPLC analytical column. Fluorescence end-point detection was used to identify TRP.

\section{Behavioural measures}

We assessed the effects of ATD on Information Sampling Task performance using repeated-measures analysis of variance (ANOVA; PASW 18, Chicago, IL) of condition (Free, Costly) by treatment (ATD, placebo). The effects of individual differences in BDI and BIS scores on task performance were examined with simple linear regressions. Interactions between treatment and BDI/BIS/BARnp scores were tested using repeated-measures analysis of covariance. Condition order, treatment order, and gender were initially included as between-subjects factors and dropped from subsequent analyses when non-significant.
We report two-tailed $P$-values. Greenhouse-Geisser corrections were applied when the sphericity assumption was violated. The data in the figures represent raw data.

\section{Results}

Biochemical measures

The ATD procedure resulted in significant reductions in both plasma TRP levels and the TRP: $L$ LNAA ratio. A repeated-measures ANOVA revealed a significant two-way interaction between treatment (ATD, placebo) and time point (baseline, $+5.5 \mathrm{~h}$ ), resulting from significant reductions in total TRP levels $(F=75.787, P<0.0001)$ and the TRP: $\Sigma$ LNAA ratio $(F=46.358, P<0.0001), 5.5 \mathrm{~h}$ following ATD relative to placebo. Simple effects analyses showed a significant decrease in plasma TRP levels $(T=4.262, P<$ 0.0001 ) on the ATD session, averaging $56 \%$. There was also a significant decrease in TRP: $\Sigma$ LNAA ratios $(T=$ $10.641, P<0.001$ ) on the ATD session, averaging $77 \%$. On the placebo session, plasma TRP levels increased by an average of $54 \%(T=-5.840, P<0.0001)$, but there was no significant change in TRP: $\Sigma$ LNAA ratios $(T=-1.220, P=$ $0.232)$.

\section{Self-report mood}

We examined the effects of time, treatment, and their interaction on PANAS positive and negative affect (Table 1). For positive affect, there were no significant effects of time ( $F=1.691, P=0.203)$, treatment $(F=0.573, P=0.455)$, or time $\times$ treatment interaction $(F=2.009, P=0.167)$. For negative affect, there was a decrease over time in both sessions (main effect of time, $F=6.4, P=0.021$ ), but there was no significant effect of treatment $(F=0.347, P=0.563)$ or treatment $\times$ interaction $(F=0.793, P=0.380)$.

Information Sampling Task performance

\section{Information sampling}

The degree of information sampling was assessed using the dependent measure $P$ (correct) at decision, which was highly correlated with the number of boxes opened but
Table 1 Effects of time and treatment on self-report mood scores. Results are reported as mean ratings (with standard errors); $\mathrm{T} 1=$ baseline, $\mathrm{T} 2=$ pretesting

\begin{tabular}{llllll}
\hline & \multicolumn{2}{l}{ ATD } & & \multicolumn{2}{l}{ PLA } \\
\cline { 2 - 3 } \cline { 5 - 6 } & T1 & & T2 & & T1 \\
\hline Positive affect & $3.912(0.177)$ & $3.618(0.167)$ & & $1.237(0.093)$ & $1.142(0.061)$ \\
Negative affect & $3.692(0.196)$ & $3.654(0.182)$ & & $1.216(0.06)$ & $1.084(0.04)$ \\
\hline
\end{tabular}


provided a more sensitive measure of the information available at the time of decision (see "Methods'). As expected, information sampling was related to accuracy. $P$ (correct) was significantly correlated with the number of correct judgments in both the Free condition (ATD: $r=$ 0.737, $P<0.001$; PLA: $r=0.590, P<0.005)$ and the Costly condition (ATD: $r=0.900, P<0.001$, PLA: $r=0.832, P<$ 0.001 ), and as these measures were correlated, we focus our analysis on the $P$ (correct) variable.

Repeated-measures ANOVA revealed a significant main effect of condition on information sampling $(F=$ 79.946, $P<0.0001)$ such that participants sampled less information in the Costly condition compared to the Free condition. The main effect of treatment was not significant $(F=1.783, P=0.197)$, but there was a significant condition $\times$ treatment interaction $(F=4.945, P=.038)$. The condition $\times$ treatment interaction remained significant when including treatment order in the model as a betweensubjects factor $(F=5.577, P=0.029)$. Treatment order was not itself significant nor did it interact significantly with any other factor (all $P>0.136$ ). Post-hoc paired $t$-tests conducted on the Free and Costly conditions separately showed that ATD tended to increase information sampling in the Costly condition $(t=1.991, P=0.060)$ but not in the Free condition $(t=-0.143, P=0.888)$. Figure 2 shows the average information sampled per trial as a function of condition and treatment.

To examine the relationship between individual differences in depressive symptomology and information sampling, we computed correlations between BDI scores and information sampling (indexed by $P($ correct)) in the Free and Costly conditions, both averaged across treatments and in the placebo session only. BDI scores were positively correlated with information sampling in the Costly condition ( $r=0.527, P=0.014$; PLA only: $r=0.510, P=0.018$; Fig. 3a). This correlation was not significant in the Free condition ( $r=0.365, P=0.103$; PLA only: $r=0.368, P=$ 0.100 ; Fig. 3b), although the coefficients for the Free and

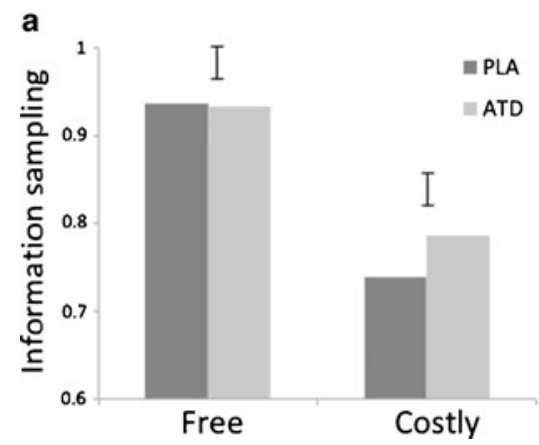

Fig. 2 a The mean information sampled per trial (defined as $P$ (correct) at decision) as a function of condition (Free, Costly) and treatment [placebo (PLA), ATD]. b The mean change in information
Costly conditions were not significantly different from one another (Steiger's $Z=0.64, P=0.52$ ).

We conducted the same analysis on the BIS scores. Here we found the reverse pattern: BIS scores were positively correlated with information sampling in the Free condition ( $r=0.583, P=0.006$; PLA only: $r=0.537, P=0.012$; Fig. $4 \mathrm{a})$ but not significantly correlated with information sampling in the Costly condition ( $r=0.146, P=0.527$; PLA only: $r=$ $0.091, P=0.695$; Fig. 4b). These two correlations were significantly different from one another at the trend level (Steiger's $Z=1.65, P=0.098)$. The observation that individual differences in the Free condition were systematically related to trait inhibition levels may potentially mitigate against a ceiling effect in the Free condition, although we acknowledge that a ceiling effect in the Free condition cannot be ruled out completely.

Meanwhile, there was no relationship between selfreported impulsivity (BARnp) and information sampling, in either the Free condition $(r=0.328, P=0.147$; PLA only: $r=0.073, P=0.755)$ or the Costly condition $(r=-0.116, P=$ 0.619 ; PLA only: $r=-0.170, P=0.461$ ).

Despite the non-significant correlations between our three self-report measures, as a further precaution against the relatively small sample size, we computed a series of partial correlations to verify that BDI and BIS scores exerted independent effects on information sampling. BDI scores were significantly correlated with information sampling in the Costly condition when controlling for BIS scores $(r=0.511, P=0.021$; PLA only: $r=0.507, P=0.023)$ and BARnp scores $(r=0.536, P=0.015$; PLA only: $r=$ $0.526, P=0.017)$. Similarly, BIS scores were significantly correlated with information sampling in the Free condition when controlling for BDI scores $(r=0.538, P=0.014$; PLA only: $r=0.487, P=0.030)$ and BARnp scores $(r=0.531, P=$ 0.016; PLA only: $r=0.546, P=0.013$ ).

Because BDI and BIS scores exerted significant effects on information sampling, we further examined whether these effects interacted with those of ATD. When including

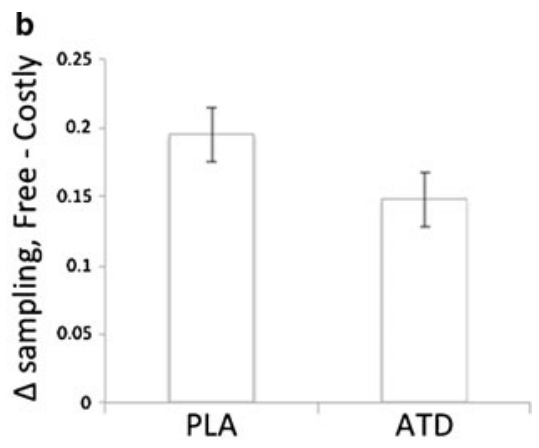

sampling from the Free condition to the Costly condition as a function of treatment. Error bars represent the standard error of the difference between treatments 

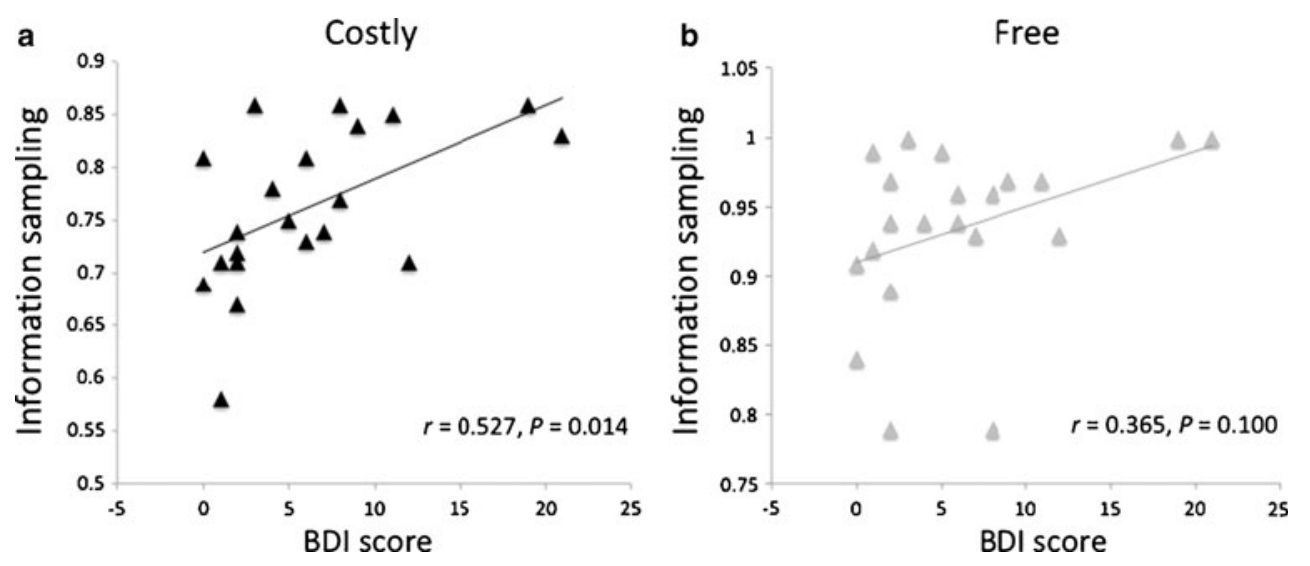

Fig. 3 Correlations between BDI scores and information sampling ( $P$ (correct) at decision) in the Costly condition (a) and Free condition (b), averaged across treatment conditions

BDI scores as a covariate in a repeated-measures ANOVA, we found significant effects of condition $(F=79.946, P<$ $0.001)$, condition-by-treatment $(F=4.945, P=0.038)$, and BDI $(F=9.837, P=0.005)$ on information sampling, but no significant interactions between treatment and BDI (all $P>$ 0.425). We observed similar results for BIS: including BIS scores as a covariate, there were significant effects of condition $(F=84.525, P<0.001)$, condition-by-treatment $(F=4.782, P=0.041)$, and BIS $(F=4.729, P=0.043)$ but no significant interactions between treatment and BIS (all $P>$ $0.729)$.

\section{Points scored}

Despite differences in strategy between conditions and treatments, participants earned the same number of points, on average, in both conditions and both treatments. Repeated-measures ANOVA showed no significant effects of condition $(F=0.482, P=0.496)$, treatment $(F=0.133, P=$ $0.720)$, or their interaction $(F=0.255, P=0.620)$ on points scored. None of the self-report measures (BDI, BIS, and
BARnp) significantly correlated with points scored in either the Free or Costly conditions (all $p>0.164$ ).

\section{Discussion}

We examined the effects of ATD on free and costly information sampling in the context of a reflection impulsivity task. ATD appeared to selectively disinhibit the suppressive effects of small local losses on information sampling behaviour; it increased costly information sampling whilst leaving free information sampling unaffected.

Our results are consistent with a recent theoretical model that characterises 5 -HT as pruning a tree of potential decisions, eliminating options expected to lead to aversive outcomes (Dayan, 2008). Here, we clarify this prediction by proposing that 5 -HT promotes reflexive avoidance of relatively immediate aversive outcomes, potentially at the expense of more globally construed future losses. Our findings complement those of a recent study showing that ATD increased the tendency to accept small losses rather
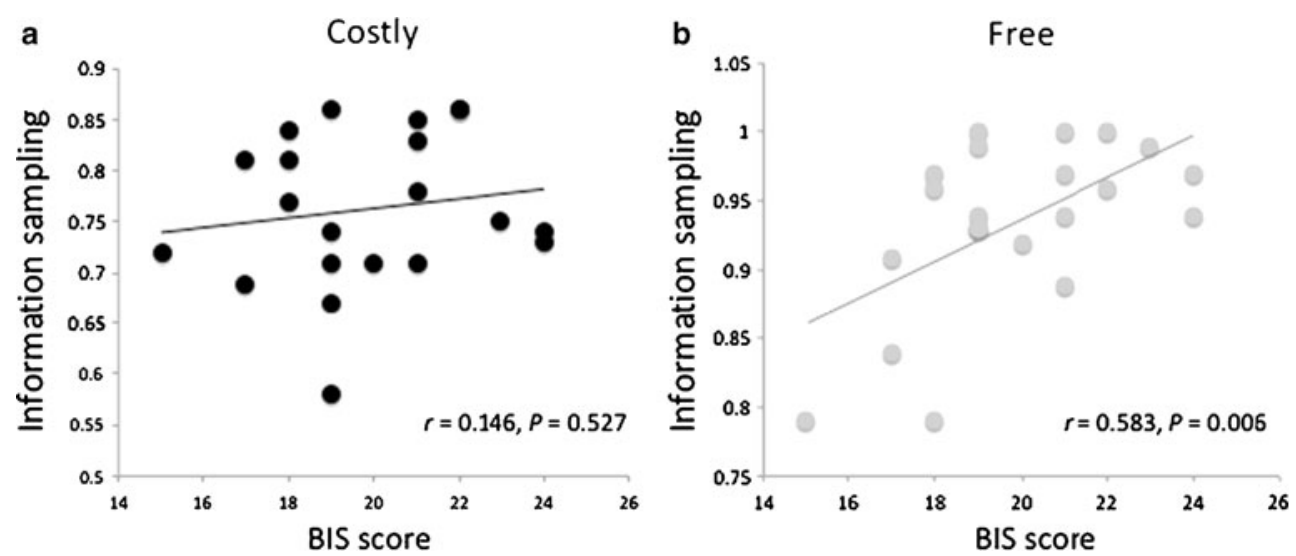

Fig. 4 Correlations between BIS scores and information sampling ( $P$ (correct) at decision) in the Costly condition (a) and Free condition (b), averaged across treatment conditions 
than risk larger losses to recover them in a 'loss-chasing' gambling context (Campbell-Meiklejohn et al., 2011). A focus on avoiding immediate losses could be adaptive under stressful conditions. Indeed, acute stressors activate the 5-HT releasing neurons of the raphé nuclei (Joëls and Baram, 2009; Takase et al., 2004), which could promote inhibition of locally costly actions. However, when stressors are controllable (for example, by instrumental escape actions), 5-HT release is inhibited (Amat et al., 2005); this could 'release' actions that may be costly in the short-term but lead ultimately to the avoidance of more global threats.

Previous studies using the Information Sampling Task suggested that subjects sampled less information in the Costly condition (relative to the Free condition) in order to maximise rewards (Clark et al., 2006; Clark et al., 2009). In the current study, we suggest an alternative construal: that subjects sample less information in the Costly condition to avoid losing potential winnings. These motivational explanations need not be mutually exclusive; indeed, a recent study reported similar activations in the striatum when subjects emitted actions to win money, and when they emitted actions to avoid losing money (Guitart-Masip et al., 2011). Interpreting our results in terms of loss avoidance is further bolstered by our analysis of the self-report data. Sub-clinical depressive and anxious symptoms exerted distinct and independent effects on information sampling and did not interact with the effects of ATD. Depressive symptoms (BDI scores) influenced behaviour in a similar direction as ATD, with more highly depressive subjects showing increased costly information sampling (although we note that this correlation did not differ significantly from the correlation between BDI scores and free information sampling). This finding fits with the observation that depression is believed to be associated with decreased 5HT levels, and ATD has been reported to induce depressive symptoms in people with a history of depression (Delgado et al., 1990). Reduced avoidance of potentially aversive outcomes, putatively associated with impaired 5-HT function, could be a risk factor for clinical depression (Dayan, 2008). This idea is supported by studies showing that depression is associated with increased cognitive engagement with aversive stimuli (Joormann and Gotlib, 2006; Harmer et al., 2009) and that treatment with SSRIs can reduce this negative cognitive bias (Harmer et al., 2009). Moreover, these findings are in line with observations that depressed patients and dysphoric subjects can show a tendency to ruminatively analyse their symptoms (NolenHoeksema, 2000) - a kind of 'costly information sampling.' It would be worthwhile to examine costly information sampling behaviour in depressed patients both on and off medication or repeatedly over the course of treatment. Increased sensitivity to the effects of cost on information sampling could be an early predictor of response to treatment with antidepressants that may be detectable sooner than changes in depressive symptoms, just as antidepressant-induced changes in negative cognitive biases occur earlier than changes in mood (Harmer, 2008).

Meanwhile, dispositional anxiety (BIS scores) was positively correlated with free information sampling but was unrelated to costly information sampling (although we note that the difference in correlations was significant only at the trend level). This finding suggests that anxious individuals are sensitive to potential losses at both the local and the global levels. When information sampling is free, increased sensitivity to potential global losses would lead to greater information sampling to avoid those losses; but when information sampling is costly, avoidance of losses at the local level could antagonise avoidance of losses at the global level. Previous studies have associated BIS scores with enhanced sensitivity to losses and threat (Perkins et al., 2010; Field, 2006; Smillie et al., 2007). The current study extends these findings to a setting involving decisionmaking under conflict and suggests that the cost of information sampling moderates the influence of anxiety on decision-making.

These findings also add to a growing literature indicating a complex relationship between 5-HT and impulsivity. In the current study, we examined the effect of ATD on 'reflection impulsivity' or the tendency to make decisions before gathering sufficient or appropriate information (Kagan, 1966). Contrary to a simple account linking reduced 5-HT function with increased impulsivity, in the current study, there was no overall main effect of ATD on information sampling, and ATD tended to reduce reflection impulsivity by attenuating the impact of cost on information sampling. In addition, there was no significant relationship between trait impulsivity (BARnp) and information sampling suggesting that variation in behaviour in the current study depended primarily on differences in aversive processing. Rather than promoting self-control in general, 5-HT more likely regulates the effects of aversive predictions on cognition and behaviour, which can result in behavioural inhibition (when behaviour leads to aversive outcomes) and also reduced cognitive reflection (when thoughts lead to aversive feelings) (Crockett et al., 2009; Dayan, 2008).

The present findings are also relevant for understanding the cognitive effects of drugs of abuse that target different neurotransmitter systems. Using the same Information Sampling Task as in the current study, previous studies have shown increased reflection impulsivity (i.e., reduced information sampling) in current and former users of amphetamines, opiates, and cannabis (Clark et al., 2006; Clark et al., 2009). Intriguingly, however, current and former users of MDMA ('ecstasy') did not differ from controls in reflection impulsivity in the Information Sampling Task, 
despite showing elevated self-reported impulsivity (Clark et al., 2009). Our results may help to explain this puzzling finding. As MDMA has neurotoxic effects on 5-HT neurons (Gouzoulis-Mayfrank and Daumann, 2006), current and former MDMA users may have reduced 5-HT neurotransmission, which could produce behavioural effects similar to ATD. In our study, ATD tended to reduce reflection impulsivity; thus, neurotoxic effects of MDMA could have counteracted the effects of trait-level impulsivity on information sampling in MDMA users.

In sum, we found that temporarily disrupting 5-HT function in humans with an ATD manipulation attenuated the impact of cost on information sampling behaviour. These results suggest that 5-HT directs reflexive avoidance of immediate aversive outcomes and imply that considering both global and local construals of threat is a useful construct for examining the effects of 5-HT on aversively motivated cognition and behaviour.

\section{Appendix 1: IST instructions}

The subject was instructed, "You are about to play a game for points. The game will take 10 minutes to complete. It consists of two parts and on each part there will be 10 gos. On every go, you will be able to see 25 boxes on the screen. Initially, the boxes will all be greyed out, but when you pick a box, it will reveal itself to be one of two colours. Your task is to decide which colour you think is in the majority. It is entirely up to you how many boxes you open before making your decision. When you have made your decision, you should touch that colour panel at the bottom of the screen." These instructions were read to the subject during a practice trial, during which 100 points were available to win or lose for a correct or wrong response. This practice trial was identical to the Fixed Win condition, for which it was further reiterated, "You will win 100 points if you pick the correct colour, regardless of how many boxes you open, and you can open as many boxes as you wish. You will lose 100 points if you get it wrong. Try to win as many points as you can." In the Decreasing Win condition, subjects were instructed, "On these gos, the amount you can win will drop by 10 points with every box you uncover, therefore the earlier you make your decision the more points you will win if you are right. If you are wrong, you will lose 100 points regardless of when you make your decision. Try to win as many points as you can."

Acknowledgements We thank the staff at the Wellcome Trust Clinical Research Facility, O. Robinson and J. Dowson for their assistance. This work was supported by a JT McDonnell Network grant and was completed within the University of Cambridge
Behavioural and Clinical Neuroscience Institute, funded by a joint award from the Medical Research Council and the Wellcome Trust. The Information Sampling Task is licensed to Cambridge Cognition Ltd. LC and TWR consult for Cambridge Cognition Ltd. LJS and MDC declare no conflict of interest.

\section{References}

Amat J, Baratta MV, Paul E, Bland ST, Watkins LR, Maier SF (2005) Medial prefrontal cortex determines how stressor controllability affects behavior and dorsal raphe nucleus. Nat Neurosci 8:365371

Ardis TC, Cahir M, Elliott JJ, Bell R, Reynolds GP, Cooper SJ (2009) Effect of acute tryptophan depletion on noradrenaline and dopamine in the rat brain. J Psychopharmacol 23:51-55

Beck AT, Steer RA, Brown GK (1996) Manual for Beck Depression Inventory-II. Psychological Corporation, San Antonio, Texas

Biggio G, Fadda F, Fanni P, Tagliamonte A, Gessa GL (1974) Rapid depletion of serum tryptophan, brain tryptophan, serotonin and 5hydroxyindoleacetic acid by a tryptophan-free diet. Life Sci 14:1321-1329

Boureau Y, Dayan P (2011) Opponency revisited: competition and cooperation between dopamine and serotonin. Neuropsychopharmacology 36(1):74-97

Campbell-Meiklejohn D, Wakeley J, Herbert V, Cook J, Scollo P, Kar Ray M, Selvaraj S, Passingham RE, Cowen P, Rogers RD (2011) Serotonin and dopamine play complementary roles in gambling to recover losses. Neuropsychopharmacology 36(2):402-10

Carpenter LL, Anderson GM, Pelton GH, Gudin JA, Kirwin PD, Price LH, Heninger GR, McDougle CJ (1998) Tryptophan depletion during continuous CSF sampling in healthy human subjects. Neuropsychopharmacology 19:26-35

Carver CS, White TL (1994) Behavioral inhibition, behavioral activation, and affective responses to impending reward and punishment: the BIS/BAS scales. J Pers Soc Psychol 67:319-319

Cervo L, Mocaër E, Bertaglia A, Samanin R (2000) Roles of 5-HT(1A) receptors in the dorsal raphe and dorsal hippocampus in anxiety assessed by the behavioral effects of 8-OH-DPAT and S 15535 in a modified Geller-Seifter conflict model. Neuropharmacology 39:1037-1043

Clark L, Roiser JP, Robbins TW, Sahakian BJ (2009) Disrupted 'reflection' impulsivity in cannabis users but not current or former ecstasy users. J Psychopharmacol 23:14-22

Clark L, Robbins TW, Ersche KD, Sahakian BJ (2006) Reflection impulsivity in current and former substance users. Biol Psychiatry 60:515-522

Clark L, Roiser JP, Cools R, Rubinsztein DC, Sahakian BJ, Robbins TW (2005) Stop signal response inhibition is not modulated by tryptophan depletion or the serotonin transporter polymorphism in healthy volunteers: implications for the 5-HT theory of impulsivity. Psychopharmacology (Berl) 182:570-578

Cools R, Nakamura K, Daw ND (2011) Serotonin and dopamine: unifying affective, activational, and decision functions. Neuropsychopharmacology 36(1):98-113

Crockett MJ, Clark L, Robbins TW (2009) Reconciling the role of serotonin in behavioral inhibition and aversion: acute tryptophan depletion abolishes punishment-induced inhibition in humans. J Neurosci 29:11993-11999

Crockett MJ, Clark L, Tabibnia G, Lieberman MD, Robbins TW (2008) Serotonin modulates behavioral reactions to unfairness. Science 320:1739

Dayan P (2008) The role of value systems in decision making. In better than conscious? In: Engel C, Singer W (eds) Implications for performance and institutional analysis. MIT Press 
Deakin JF (2003) Depression and antisocial personality disorder: two contrasting disorders of 5HT function. J Neural Transm Suppl:79-93.

Deakin JFW, Graeff FG (1991) 5-HT and mechanisms of defence. J Psychopharmacol 5:305-315

Delgado PL, Charney DS, Price LH, Aghajanian GK, Landis H, Heninger GR (1990) Serotonin function and the mechanism of antidepressant action. Reversal of antidepressant-induced remission by rapid depletion of plasma tryptophan. Arch Gen Psychiatry 47:411-418

Dhar R, Kim EY (2007) Seeing the forest or the trees: implication of construal level theory for consumer choice. J Consum Psychol 17 (2):96-100

Dolan M, Anderson IM, Deakin JF (2001) Relationship between 5-HT function and impulsivity and aggression in male offenders with personality disorders. Br J Psychiatry 178:352-359

Evenden JL (1999) Varieties of impulsivity. Psychopharmacology (Berl) 146:348-361

Field AP (2006) The behavioral inhibition system and the verbal information pathway to children's fears. J Abnorm Psychol 115:742-752

Geller I, DeMarco AO, Seifter J (1960) Delayed effects of nicotine on timing behavior in the rat. Science 131:735-737

Gessa GL, Biggio G, Fadda F, Corsini GU, Tagliamonte A (1974) Effect of the oral administration of tryptophan-free amino acid mixtures on serum tryptophan, brain tryptophan and serotonin metabolism. J Neurochem 22:869-870

Gouzoulis-Mayfrank E, Daumann J (2006) Neurotoxicity of methylenedioxyamphetamines (MDMA; ecstasy) in humans: how strong is the evidence for persistent brain damage? Addiction 101:348-361

Graeff FG (2002) On serotonin and experimental anxiety. Psychopharmacology (Berl) 163:467-476

Graeff FG, Schoenfeld RI (1970) Tryptaminergic mechanisms in punished and nonpunished behavior. J Pharmacol Exp Ther 173:277-283

Guitart-Masip M, Fuentemilla L, Bach DR, Huys QJ, Dayan P, Dolan RJ, Duzel E (2011) Action dominates valence in anticipatory representations in the human striatum and dopaminergic midbrain. J Neurosci 31:7867-7875

Harmer CJ (2008) Serotonin and emotional processing: does it help explain antidepressant drug action? Neuropharmacology 55:1023-1028

Harmer CJ, O'Sullivan U, Favaron E, Massey-Chase R, Ayres R, Reinecke A, Goodwin GM, Cowen PJ (2009) Effect of acute antidepressant administration on negative affective bias in depressed patients. Am J Psychiatry 166:1178-1184

Joëls M, Baram TZ (2009) The neuro-symphony of stress. Nat Rev Neurosci 10:459-466

Joormann J, Gotlib IH (2006) Is this happiness I see? Biases in the identification of emotional facial expressions in depression and social phobia. J Abnorm Psychol 115:705-714

Kagan J (1966) Reflection-impulsivity: the generality and dynamics of conceptual tempo. J Abnorm Psychol 71:17-24

Meda SA, Stevens MC, Potenza MN, Pittman B, Gueorguieva R, Andrews MM, Thomas AD, Muska C, Hylton JL, Pearlson GD (2009) Investigating the behavioral and self-report constructs of impulsivity domains using principal component analysis. Behav Pharmacol 20:390-399

Moja EA, Cipolla P, Castoldi D, Tofanetti O (1989) Dose-response decrease in plasma tryptophan and in brain tryptophan and serotonin after tryptophan-free amino acid mixtures in rats. Life Sci 44:971-976
Neumeister A (2003) Tryptophan depletion, serotonin, and depression: where do we stand? Psychopharmacol Bull 37:99-115

Nolen-Hoeksema S (2000) The role of rumination in depressive disorders and mixed anxiety/depressive symptoms. J Abnorm Psychol 109:504-511

Owens MJ, Nemeroff CB (1994) Role of serotonin in the pathophysiology of depression: focus on the serotonin transporter. Clin Chem 40:288-295

Patton JH, Stanford MS (1995) Factor structure of the Barratt impulsiveness scale. J Clin Psychol 51:768-774

Perkins AM, Cooper A, Abdelall M, Smillie LD, Corr PJ (2010) Personality and defensive reactions: fear, trait anxiety, and threat magnification. J Pers 78:1071-1090

Reynolds B, Ortengren A, Richards JB, de Wit H (2006) Dimensions of impulsive behavior: personality and behavioral measures. Personal Individ Differ 40:305-315

Rinne T, van den Brink W, Wouters L, van Dyck R (2002) SSRI treatment of borderline personality disorder: a randomized, placebo-controlled clinical trial for female patients with borderline personality disorder. Am J Psychiatry 159:2048-2054

Robbins TW, Crockett MJ (2010) Role of central serotonin in impulsivity and compulsivity: comparative studies in experimental animals and humans. In: Handbook of the behavioral neurobiology of serotonin (Muller CP, Jacobs BL, eds), pp 415-427.

Smillie LD, Dalgleish LI, Jackson CJ (2007) Distinguishing between learning and motivation in behavioral tests of the reinforcement sensitivity theory of personality. Pers Soc Psychol Bull 33:476-489

Soubrie P (1986) Reconciling the role of central serotonin neurons in human and animal behavior. Behav Brain Sci 9:364

Stancampiano R, Melis F, Sarais L, Cocco S, Cugusi C, Fadda F (1997) Acute administration of a tryptophan-free amino acid mixture decreases 5-HT release in rat hippocampus in vivo. Am J Physiol 272:R991-R994

Stevens DA, Fechter LD, Resnick O (1969) The effects of pchlorophenylalanine, a depletor of brain serotonin, on behavior: II. Retardation of passive avoidance learning. Life Sci 8:379-385

Takase LF, Nogueira MI, Baratta M, Bland ST, Watkins LR, Maier SF, Fornal CA, Jacobs BL (2004) Inescapable shock activates serotonergic neurons in all raphe nuclei of rat. Behav Brain Res 153:233-239

Thaler R (1985) Mental accounting and consumer choice. Mark Sci 4 (3): 199-214

Thiébot MH, Hamon M, Soubríe P (1982) Attenuation of inducedanxiety in rats by chlordiazepoxide: role of raphe dorsalis benzodiazepine binding sites and serotoninergic neurons. Neuroscience 7:2287-2294

Trope Y, Liberman N (2000) Temporal construal and time-dependent changes in preference. J Pers Soc Psychol 79(6):876-889

Trope Y, Liberman N (2003) Temporal construal. Psychol Rev 110:403-421

Tye NC, Everitt BJ, Iversen SD (1977) 5-Hydroxytryptamine and punishment. Nature 268:741-743

Wakslak C, Trope Y (2009) The effect of construal level on subjective probability estimates. Psychol Sci 20(1):52-58

Watson D, Clark LA, Tellegen A (1988) Development and validation of brief measures of positive and negative affect: the PANAS scales. J Pers Soc Psychol 54:1063-1070

Winstanley CA, Dalley JW, Theobald DE, Robbins TW (2004) Fractionating impulsivity: contrasting effects of central 5-HT depletion on different measures of impulsive behavior. Neuropsychopharmacology 29:1331-1343 
Wise CD, Berger BD, Stein L (1972) Benzodiazepines: anxietyreducing activity by reduction of serotonin turnover in the brain. Science 177:180-183

Wise CD, Berger BD, Stein L (1973) Evidence of -noradrenergic reward receptors and serotonergic punishment receptors in the rat brain. Biol Psychiatry 6:3-21

de Wit H, Flory JD, Acheson A, McCloskey M, Manuck SB (2007) IQ and nonplanning impulsivity are independently associated with delay discounting in middle-aged adults. Personal Individ Differ 42:111-121

Zanarini MC, Frankenburg FR, Parachini EA (2004) A preliminary, randomized trial of fluoxetine, olanzapine, and the olanzapinefluoxetine combination in women with borderline personality disorder. J Clin Psychiatry 65:903-907

Zermatten A, Van der Linden M, d'Acremont M, Jermann F, Bechara A (2005) Impulsivity and decision making. J Nerv Ment Dis 193:647-650 